\title{
Boundary Stabilization of a Nonlinear Viscoelastic Equation with Interior Time-Varying Delay and Nonlinear Dissipative Boundary Feedback
}

\author{
Zaiyun Zhang, ${ }^{1,2}$ Jianhua Huang, ${ }^{1}$ Zhenhai Liu, ${ }^{3}$ and Mingbao Sun ${ }^{2}$ \\ ${ }^{1}$ College of Science, National University of Defense Technology, Changsha, Hunan 410083, China \\ ${ }^{2}$ School of Mathematics, Hunan Institute of Science and Technology, Yueyang, Hunan 414006, China \\ ${ }^{3}$ School of Mathematics and Computer Science, Guangxi University for Nationalities, Nanning, Guangxi 530006, China \\ Correspondence should be addressed to Zaiyun Zhang; zhangzaiyun1226@126.com
}

Received 22 January 2014; Revised 2 May 2014; Accepted 16 May 2014; Published 9 June 2014

Academic Editor: Chuangxia Huang

Copyright ( 2014 Zaiyun Zhang et al. This is an open access article distributed under the Creative Commons Attribution License, which permits unrestricted use, distribution, and reproduction in any medium, provided the original work is properly cited.

\begin{abstract}
We investigate a nonlinear viscoelastic equation with interior time-varying delay and nonlinear dissipative boundary feedback. Under suitable assumptions on the relaxation function and time-varying delay effect together with nonlinear dissipative boundary feedback, we prove the global existence of weak solutions and asymptotic behavior of the energy by using the Faedo-Galerkin method and the perturbed energy method, respectively. This result improves earlier ones in the literature, such as Kirane and SaidHouari (2011) and Ammari et al. (2010). Moreover, we give an positive answer to the open problem given by Kirane and Said-Houari (2011).
\end{abstract}

\section{Introduction}

In this paper, we consider the global existence and asymptotic behavior of a nonlinear viscoelastic equation with interior time-varying delay and nonlinear dissipative boundary feedback as follows:

$$
\begin{gathered}
u_{t t}-\Delta u+\int_{0}^{t} h(t-s) \Delta u(s) d s+a u_{t}(x, t-\tau(t))=0, \\
x \in \Omega, \quad t>0, \\
u(x, t)=0, \quad \text { on } \Gamma_{0} \times(0, \infty), \\
\frac{\partial u}{\partial \nu}+g\left(u_{t}(x, t)\right)=0, \quad \text { on } \Gamma_{1} \times[0, \infty), \\
u(x, 0)=u_{0}(x), \quad u_{t}(x, 0)=u_{1}(x), \quad x \in \Omega, \\
u_{t}(x, t-\tau(t))=f_{0}(x, t), \quad x \in \Omega,-\tau(0) \leq t \leq 0,
\end{gathered}
$$

where $\Omega$ is a bounded domain of $R^{n}(n \geq 1)$ with a smooth boundary $\partial \Omega$ of $C^{2}, a$ is a positive real constant, $\tau(t)>0$ represents the time-varying delay effect and the initial data $u_{0}, u_{1}, f_{0}$ are given functions belonging to suitable spaces, $h(t)$ is a positive function that represents the kernel of the memory term, $g\left(u_{t}\right)$ is nonlinear dissipative boundary feedback, and $f_{0}, h, g$ satisfy suitable assumptions (see in Section 2).

This model appears in viscoelasticity (see $[1,2])$. In the case of velocity-dependent material density (i.e., $\rho=0$ ) as well as presence of $\mu_{2}=0$ and in the absence of the memory effect (i.e., $g=0$ ), (1) reduces to the wave equation. There is large literature on the global existence and uniform stabilization of wave equations. We refer the readers to [3-5]. It is worth mentioning that Zhang and Miao [3] considered the nonlinear wave equation with dissipative term and boundary damping

$$
\begin{gathered}
u_{t t}-\Delta u+a(x) u_{t}+f(u)=0, \quad \text { in } \Omega \times[0, \infty), \\
u=0, \quad \text { on } \Gamma_{1} \times[0, \infty), \\
\frac{\partial u}{\partial v}+g\left(u_{t}\right)=0, \text { on } \Gamma_{0} \times[0, \infty), \\
u(x, 0)=u^{0}(x), \quad u_{t}(x, 0)=u^{1}(x), \quad \text { in } \Omega,
\end{gathered}
$$


and they proved the existence and uniform decay of strong and weak solutions by using the Glerkin method and the multiplier technique, respectively. Later on, Zhang et al. [4] improved earlier ones in [3]. More precisely, they investigated the global existence and uniform stabilization of generalized dissipative Klein-Gordon equation with boundary damping

$$
\begin{gathered}
u_{t t}-\Delta u+b(x) u_{t}+f(u)+h(\nabla u)=0, \quad \text { in } \Omega \times[0, \infty), \\
u=0, \quad \text { on } \Gamma_{1} \times[0, \infty), \\
\frac{\partial u}{\partial v}+g\left(u_{t}\right)=0, \quad \text { on } \Gamma_{0} \times[0, \infty), \\
u(x, 0)=u^{0}(x), \quad u_{t}(x, 0)=u^{1}(x), \quad \text { in } \Omega,
\end{gathered}
$$

and they proved the existence and uniform decay of strong and weak solutions by using the nonlinear semigroup method, the perturbed energy method, and the multiplier technique. Quite recently, Cavalcanti et al. [6] considered the following model:

$$
\begin{gathered}
u_{t t}-\Delta_{\mathscr{M}} u+a(x) g\left(u_{t}\right)=0, \quad \text { on } \mathscr{M} \times(0, \infty), \\
u(x, 0)=u^{0}(x), \quad u_{t}(x, 0)=u^{1}(x), \quad \text { for } x \in \mathscr{M},
\end{gathered}
$$

where $\mathscr{M}$ is a smooth oriented embedded compact surface without boundary in $R^{3}$ and $\Delta_{\mathscr{M}}$ is the Laplace-Beltrami operator on manifold $\mathscr{M}$; furthermore, they obtained explicit and optimal decay rates of the energy. Later on, Cavalcanti et al. [7] extended the result for $n$-dimensional compact Riemannian manifolds $(\mathscr{M}, g)$ with boundary in two ways: (i) by reducing arbitrarily the region where the dissipative effect lies (this gives us a totally sharp result with respect to the boundary measure and interior measure where the damping is effective) and (ii) by controlling the existence of subsets on the manifold that can be left without any dissipative mechanism, namely, a precise part of radially symmetric subsets. An analogous result holds for compact Riemannian manifolds without boundary.

In the case $\rho=0$ and in the absence of delay (i.e., $\mu_{2}=0$ ), there is large literature on the existence and decay of nonlinear viscoelastic equation during the past decades. In [8], Cavalcanti et al. considered the exponential decay for the solution of viscoelastic wave equation with localized damping

$$
\begin{array}{r}
\left|u_{t}\right|^{\rho} u_{t t}-\Delta u+\int_{0}^{t} g(t-s) \Delta u(s) d s+a(x) u_{t}+u|u|^{r}=0, \\
x \in \Omega, \quad t>0 .
\end{array}
$$

Under the condition that $a(x) \geq a_{0}>0$ on $\omega \subset \Omega$, with $\omega$ satisfying some geometry restrictions and

$$
-\xi_{1} g(t) \leq g^{\prime}(t) \leq-\xi_{2} g(t), \quad t \geq 0,
$$

they proved an exponential decay result for the energy. Berrimi and Messaoudi [9] improved Cavalcanti's result by introducing a differential functional which allowed to weaken the conditions on both $a(x)$ and $g$. In [10], Cavalcanti and Oquendo studied

$$
\begin{gathered}
\left|u_{t}\right|^{\rho} u_{t t}-k_{0} \Delta u+\int_{0}^{t} \operatorname{div}[a(x) g(t-s) \nabla u(s)] d s \\
+b(x) h\left(u_{t}\right)+f(u)=0, \quad x \in \Omega, t>0 .
\end{gathered}
$$

Under some geometric restrictions on $\omega$ and assuming that

$$
\begin{gathered}
a(x) \geq a_{0}>0, \quad \forall x \in \omega, \\
-\xi_{1} g(t) \leq g^{\prime}(t) \leq-\xi_{2} g(t), \quad t \geq 0, \\
a(x)+b(x) \geq \rho>0, \quad \forall x \in \Omega,
\end{gathered}
$$

they established an exponential stability for the relaxation function $g$ decaying exponentially and $h$ linear and polynomial stability for $g$ decaying polynomially and $h$ nonlinear. It is worth mentioning that Zhang et al. [11] studied the following initial boundary value problem:

$$
\begin{gathered}
u_{t t}+A u+\int_{0}^{t} g(t-s) A u d s=0 \quad \text { in } \Omega \times(0, \infty), \\
u=0 \quad \text { on } \Gamma \times(0, \infty) \\
u(0)=u_{0}, \quad u_{t}(0)=u_{1} .
\end{gathered}
$$

Furthermore, they showed that the solutions of (9) decay uniformly in time, with rates depending on the rate of decay of the kernel $g$. More precisely, the solution decays exponentially to zero provided that $g$ decays exponentially to zero. When $g$ decays polynomially, we show that the corresponding solution also decays polynomially to zero with the same rate of decay. For other related works, we refer the readers to [12-21] and the references therein.

On the other hand, concerning the study of the following nonlinear viscoelastic equation with memory, there are a substantial number of contributions:

$$
\left|u_{t}\right|^{\rho} u_{t t}-\Delta u+\int_{0}^{t} g(t-s) \Delta u(s) d s+F\left(u, u_{t}, u_{t t}\right)=0 .
$$

Recently, Han and Wang [22] investigated the following problem:

$$
\left|u_{t}\right|^{\rho} u_{t t}-\Delta u-\Delta u_{t t}+\int_{0}^{t} g(t-s) \Delta u(s) d s=b|u|^{p-2} u .
$$

By introducing a new functional and using potential well method, the authors established the global existence and uniform decay if the initial data are in a suitable stable set. Cavalcanti et al. [23] studied a related problem with strong damping as follows:

$$
\left|u_{t}\right|^{\rho} u_{t t}-\Delta u-\Delta u_{t t}+\int_{0}^{t} g(t-s) \Delta u(s) d s-\gamma \Delta u_{t}=0 .
$$

By assuming $0<\rho \leq 2 /(n-2)$, if $n \geq 3$ or $\rho>0$ and if $n=1,2$ and $g(t)$ decays exponentially, they established that the global 
existence resulted for $\gamma \geq 0$ and the exponential decay of the energy for $\gamma>0$. This result has been extended to a situation $\gamma=0$ by Messaoudi and Tatar [24] and exponential decay and polynomial decay results have been shown in the absence as well as presence of a source term. Later on, inspired by the ideas of [25-27], Han and Wang [22] investigated the general decay of solutions of energy for the nonlinear viscoelastic equation

$$
\left|u_{t}\right|^{\rho} u_{t t}-\Delta u-\Delta u_{t t}+\int_{0}^{t} g(t-s) \Delta u(s) d s+u_{t}\left|u_{t}\right|^{k}=0
$$

In recent years, the control of partial differential equation with time delay effects has become an active area of research; see, for instance, [28, 29] and the references therein. The presence of delay may be a source of instability. For instance, it was proved in [30-34] that an arbitrarily small delay may destabilize a system which is uniformly asymptotically stable in the absence of delay unless additional conditions or control terms have been used. In [32], Nicaise and Pignotti examined (1) with $\rho=0, g \equiv 0, \mu_{1}>0, \mu_{2}>0$, and $\tau(t)=\tau$ being a constant delay in the case of mixed homogeneous Dirichlet-Neumann boundary conditions, under a geometric condition on the Neumann part of the boundary. More precisely, they investigated the following system with linear frictional damping term and internal constant delay:

$$
\begin{array}{r}
u_{t t}(x, t)-\Delta u(x, t)+\mu_{1} u_{t}(x, t)+\mu_{2} u_{t}(x, t-\tau)=0, \\
x \in \Omega, \quad t>0 \\
u(x, t)=0, \quad x \in \Gamma_{0}, t>0 \\
\frac{\partial u}{\partial v}(x, t)=0, \quad x \in \Gamma_{1}, t>0
\end{array}
$$

or with boundary constant delay

$$
\begin{array}{r}
u_{t t}(x, t)-\Delta u(x, t)=0, \quad x \in \Omega, t>0, \\
u(x, t)=0, \quad x \in \Gamma_{0}, t>0, \\
\frac{\partial u}{\partial v}(x, t)+\mu_{1} u_{t}(x, t)+\mu_{2} u_{t}(x, t-\tau)=0, \\
x \in \Gamma_{1}, \quad t>0 .
\end{array}
$$

In the presence of delay $\left(\mu_{2}>0\right)$, Nicaise and Pignotti [32] examined systems (14) and (15) and proved under the assumptions $\mu_{2}<\mu_{1}$ that the energy is exponentially stable. Otherwise, they constructed a sequence of delays for which the corresponding solution is instable. The main approach used there is an observability inequality together with a Carleman estimate. See also [35] for treatment to these problems in more general abstract form and [36] for analogous results in the case of boundary time-varying delay. We also recall the result by Nicaise et al. [36], where the researchers proved the same result as in [32] for the one space dimension by applying the spectral analysis approach. Recently, Kirane and Said-Houari [37] considered (1) with $\rho=0, \mu_{1}>0, \mu_{2}>0$, and $\tau(t) \equiv \tau$ being a constant delay in the case of the initial and Dirichlet boundary wave equation with a linear damping and a delay term as follows:

$$
\begin{gathered}
u_{t t}-\Delta u+\int_{0}^{t} g(t-s) \Delta u(s) d s+\mu_{1} u_{t}(x, t) \\
+\mu_{2} u_{t}(x, t-\tau)=0, \quad x \in \Omega, t>0, \\
u(x, t)=0, \quad x \in \partial \Omega, t>0, \\
u(x, 0)=u_{0}(x), \quad u_{t}(x, 0)=u_{1}(x), \quad x \in \Omega, \\
u_{t}(x, t-\tau)=f_{0}(x, t-\tau), \quad x \in \Omega, t \in(0, \tau) .
\end{gathered}
$$

Under an assumption between the weight of the delay term in the feedback and the weight of the term without delay, using the Faedo-Galerkin method combined with some energy estimate, they proved the global existence of (16). Also, they proved exponential decay of (16) via suitable Lyapunov functionals.

Recently, the stability of PDEs with time-varying delays was studied in [38-44]. In [40], Nicaise and Pignotti investigated the stabilization problem by interior damping of the wave equation with internal time-varying delay feedback and established exponential stability estimates by introducing suitable Lyapunov functionals, under the condition $\left|\mu_{2}\right|<$ $\sqrt{1-d} \mu_{1}$ in which the positivity of the coefficient $\mu_{1}$ is not necessary. In [41], Nicaise et al. showed the exponential stability of the heat and wave equations with time-varying boundary delay in 1-D, under the condition $0 \leq \mu_{2}<$ $\sqrt{1-d} \mu_{1}$, where $d$ is a constant such that $\tau^{\prime}(t) \leq d<1$.

The rest of the paper is organized as follows. In Section 2, we show some assumptions and state our main result. In Section 3, we present the proof of our main result. That is, we will prove the global existence by using Faedo-Galerkin method and establish the general decay result (including exponential decay and polynomial decay) by using the perturbed energy method. Finally, in Section 4, we give further remarks on this context.

\section{Some Assumptions and Main Results}

In this section, before proceeding to our analysis, we present some assumptions and state the main result. We use the standard Hilbert space $L^{2}(\Omega)$ and the Sobolev space $H_{0}^{1}(\Omega)$ with their usual scalar products and norms. Throughout this paper, $C_{i}$ is used to denote a generic positive constant from line to line.

For the relaxation function $h$, we assume that

(G1) $h(t):(0, \infty) \rightarrow(0, \infty)$ is a nonincreasing differentiable function such that

$$
1-\int_{0}^{\infty} h(s) d s=l>0 ;
$$

(G2) there exists a nonincreasing differentiable function $\zeta(t)$ such that

$$
h^{\prime}(t) \leq-\zeta(t) h^{p}(t), \quad 1 \leq p<\frac{3}{2}, t \geq 0 .
$$


We assume that $\rho$ satisfies

$$
0<\rho \leq \frac{2}{n-2}, \quad \text { if } n \geq 3 ; \quad \rho>0, \quad \text { if } n=1,2 .
$$

For the time-varying delay, we assume that there exist positive constant $\tau_{0}, \bar{\tau}$ such that

$$
0<\tau_{0} \leq \tau(t) \leq \bar{\tau}, \quad \forall t>0
$$

Furthermore, we assume that the delay satisfies

$$
\tau^{\prime}(t) \leq d<1, \quad \forall t>0,
$$

that

$$
\tau(t) \in W^{2, \infty}([0, T]), \quad \forall T>0,
$$

and that $\mu_{1}, \mu_{2}$ satisfy

$$
\left|\mu_{2}\right|<\sqrt{1-d} \mu_{1} .
$$

Remark 1. We show an example of functions satisfying (G2) as follows:

$$
\begin{gathered}
h(s)=e^{-\sigma s}, \quad p=1, \\
h(s)=\vartheta(1+s)^{-1 /(p-1)}, \quad p>1,
\end{gathered}
$$

for $\sigma, \vartheta>0$ to be chosen properly; see [2].

Remark 2. Condition $p<3 / 2$ is imposed so that $\int_{0}^{\infty} h^{2-p}(s) d s<\infty$.

Now, we are in a position to state our main results.

Theorem 3. Let (20)-(23) be satisfied and h satisfy (G2). Then, given $\left(u_{0}, u_{1}\right) \in H_{0}^{1}(\Omega) \times L^{2}(\Omega), f_{0} \in L^{2}(\Omega \times(0,1))$, and $T>0$, there exists a unique weak solution $u(x, t)$ such that

$$
\begin{aligned}
& u \in C\left(0, T ; H_{0}^{1}(\Omega)\right) \cap C^{1}\left(0, T ; L^{2}(\Omega)\right), \\
& u_{t} \in L^{2}\left(0, T ; H_{0}^{1}(\Omega)\right) \cap L^{2}((0, T) \times \Omega) .
\end{aligned}
$$

Moreover, if (20)-(23) hold and h satisfies (G1) and (G2), then there exist two positive constants $K, k$ such that for any solution of the problem (1) of the energy satisfies

$$
\begin{gathered}
\mathscr{E}(t) \leq K e^{-k t}, \quad p=1, t \geq t_{0}, \\
\mathscr{E}(t) \leq K(1+t)^{-1 /(p-1)}, \quad p>1, t \geq t_{0} .
\end{gathered}
$$

\section{Proof of the Main Result}

In this section, we will divide our proof into two steps. In Step 1, we prove the global existence of weak solutions by using Faedo-Galerkin method benefited from the ideas of $[2,3,37]$. In Step 2, we establish the general decay of energy by introducing the new energy functional and using the perturbed energy method inspired by the contributions; see, for instance, $[2-4,11,39]$.
Step 1 (global existence of weak solutions). Let $\{\omega\}_{j}^{\infty}$ be an orthogonal basis of $H_{0}^{1}(\Omega)$ with $\omega_{j}$ being the eigenfunction of the following problem:

$$
\begin{gathered}
-\Delta \omega_{j}=\lambda_{j} \omega_{j}, \quad x \in \Omega, \\
\omega_{j}=0, \quad x \in \partial \Omega .
\end{gathered}
$$

Denote $W_{n}=\operatorname{Span}\left\{\omega_{1}, \omega_{2}, \ldots, \omega_{n}\right\}$ for subspace generated by the first $n$ vectors of the basis of $\{\omega\}_{j}^{\infty}$. Then, we construct approximation of the solution $(u, z)$ as follows:

$$
\begin{gathered}
u_{n}(x, t)=\sum_{j=1}^{n} g_{j n}(t) \omega_{j}, \\
z_{n}(x, t, \rho)=\sum_{j=1}^{m} h_{j n}(t) \phi_{j}(x, \rho)
\end{gathered}
$$

and we choose two sequences $u_{0 n}$ and $u_{1 n}$ in $W_{n}$ and a sequence $z_{0 n}$ in $V_{n}$ such that $u_{0 n} \rightarrow u_{0}$ strongly in $H_{0}^{1}(\Omega), u_{1 n} \rightarrow u_{1}$ strongly in $L^{2}(\Omega)$, and $z_{0 n} \rightarrow f_{0}$ strongly in $L^{2}(\Omega \times(0,1))$. Define the sequence $\phi_{j}(x, \rho)$ as follows: $\phi_{j}(x, 0)=\phi_{j}(x)$. Then, from [37, pp 1069], we may extend $\phi_{j}(x, 0)$ by $\phi_{j}(x, \rho)$ over $L^{2}(\Omega \times(0,1))$ and denote $V_{n}=$ $\operatorname{Span}\left\{\phi_{1}, \phi_{2}, \ldots, \phi_{n}\right\}$.

To facilitate further our analysis, we introduce as in [32, 36,39 ] the new variable

$$
z(x, \theta, t)=u_{t}(x, t-\tau(t) \theta), \quad x \in \Omega, \theta \in(0,1), t>0 .
$$

Then, we get

$$
\begin{array}{r}
\tau(t) z(x, \theta, t)+\left(1-\tau^{\prime}(t) \theta\right) z_{\theta}(x, \theta, t)=0, \\
x \in \Omega, \quad \theta \in(0,1), \quad t>0 .
\end{array}
$$

Therefore, the problem (1) can be rewritten as follows:

$$
\begin{gathered}
\left|u_{t}\right|^{\rho} u_{t t}-\Delta u+\int_{0}^{t} h(t-s) \Delta u(s) d s \\
+\mu_{1} u_{t}(x, t)+\mu_{2} z(x, 1, t)=0, \quad x \in \Omega, t>0, \\
\tau(t) z(x, \theta, t)+\left(1-\tau^{\prime}(t) \theta\right) z_{\theta}(x, \theta, t)=0, \\
x \in \Omega, \quad \theta \in(0,1), \quad t>0 \\
u(x, 0)=u_{0}(x), \quad u_{t}(x, 0)=u_{1}(x), \quad x \in \Omega, \\
\quad z(x, \theta, 0)=f_{0}(x,-\tau(0)), \\
\quad \theta \in(0,1), \quad-\tau(0) \leq t \leq 0 .
\end{gathered}
$$


Hence, $\left(u_{n}(t), z_{n}(t)\right)$ are solutions to the following Cauchy problem as follows:

$$
\begin{gathered}
\int_{\Omega}\left|u_{t n}\right|^{\rho} u_{t t n} \omega_{j} d x+\int_{\Omega} \nabla u_{n} \nabla \omega_{j} d x \\
-\int_{0}^{t} h(t-s) \nabla u(s) \nabla \omega_{j} d x d s \\
+\int_{\Omega}\left[\mu_{1} u_{t n}(x, t)+\mu_{2} z_{n}(x, 1, t)\right] \omega_{j} d x=0 \\
z_{n}(x, 0, t)=u_{t n}(x, t), \\
\left(u_{n}(0), u_{t n}(0)\right)=\left(u_{0 n}, u_{1 n}\right), \\
\int_{\Omega}\left[\tau(t) z_{n t}(x, \theta, t)+\left(1-\tau^{\prime}(t) \theta\right) z_{n \theta}(x, \theta, t)\right] \phi_{j} d x=0, \\
z_{n, 0}=z_{0 n} .
\end{gathered}
$$

By standard method of ODE, we know that there exists only one local solution of the Cauchy problem (33) and (34) on some interval $\left[0, t_{n}\right), 0<t_{n}<T$, for arbitrary $T>0$; then, this solution can be extended to the whole interval $[0, T]$ by a priori estimates below.

To facilitate further our analysis, we need some notations and technical Lemmas 4 and 6. Let us first introduce some notations

$$
\begin{gathered}
(\phi \star \psi)(t)=\int_{0}^{t} \phi(t-s) \psi(s) d s \\
(\phi \diamond \psi)(t)=\int_{0}^{t} \phi(t-s)|\psi(t)-\psi(s)| d s, \\
(\phi \circ \psi)(t)=\int_{0}^{t} \phi(t-s) \int_{\Omega}|\psi(t)-\psi(s)|^{2} d x d s,
\end{gathered}
$$

with these notations; we have the following lemma given in $[2,11]$.

Lemma 4. For $\phi \in C^{1}(\mathscr{R})$ and $\psi \in H^{1}(0, T)$, one has

$$
\begin{aligned}
(\phi \star \psi)(t) \cdot \psi(t)= & -\frac{1}{2} \phi(t)\|\psi(t)\|^{2}+\frac{1}{2}\left(\phi^{\prime} \diamond \psi\right)(t) \\
- & \frac{1}{2} \frac{d}{d t}[(\phi \diamond \psi)(t) \\
& \left.-\left(\int_{0}^{t} \phi(s) d s\right)|\phi|^{2} d x\right] .
\end{aligned}
$$

Remark 5. In fact, the proof of this lemma follows by differentiating the term $g \diamond \phi$. More details are presented in $[2,11,37]$.

Lemma 6. Assuming that $v \in L^{\infty}\left(0, T ; H^{1}(\Omega)\right), h$ is a continuous function such that

$$
\int_{0}^{\infty} h^{1-\alpha}(s) d s<\infty, \quad 0 \leq \alpha \leq 1 .
$$

Then, we have

$$
\begin{aligned}
(h \circ \nabla v)(t) \leq & 2\left[\int_{0}^{t}\|\nabla v(s)\|_{2}^{2} d s+t\|\nabla v(t)\|_{2}^{2}\right]^{(p-1) / p} \\
& \times((h \circ \nabla v)(t))^{1 / p} .
\end{aligned}
$$

Proof. It suffices to observe that, for $q>1,0 \leq \alpha \leq 1$,

$$
\begin{aligned}
(h \circ \nabla v)(t)= & \int_{0}^{t} h(t-s)\|\nabla v(t)-\nabla v(s)\|_{2}^{2} d s \\
= & \int_{0}^{t} h^{(1-\alpha) / q}(t-s)\|\nabla v(t)-\nabla v(s)\|_{2}^{2 / q} h^{(q-1+\alpha) / q} \\
& \quad \times(t-s)\|\nabla v(t)-\nabla v(s)\|_{2}^{2(q-1) / q} d s .
\end{aligned}
$$

By applying Hölder inequality, we obtain

$$
\begin{aligned}
& (h \circ \nabla v)(t) \\
& \leq\left(\int_{0}^{t} h^{(1-\alpha) / q}(t-s)\|\nabla v(t)-\nabla v(s)\|_{2}^{2} d s\right)^{1 / q} \\
& \quad \times\left(\int_{0}^{t} h^{(q-1+\alpha) /(q-1)}(t-s)\|\nabla v(t)-\nabla v(s)\|_{2}^{2} d s\right)^{(q-1) / q} .
\end{aligned}
$$

Taking $q=(p-1+\alpha) /(p-1)$, we get

$$
\begin{aligned}
& (h \circ \nabla v)(t) \\
& \leq\left(\int_{0}^{t} h^{(1-\alpha)(p-1) /(p-1+\alpha)}(t-s)\right. \\
& \left.\quad \times\|\nabla v(t)-\nabla v(s)\|_{2}^{2} d s\right)^{(p-1) /(p-1+\alpha)} \\
& \quad \times\left(\int_{0}^{t} h^{p \alpha /(p-1+\alpha)}(t-s)\|\nabla v(t)-\nabla v(s)\|_{2}^{2} d s\right)^{\alpha /(p-1+\alpha)} .
\end{aligned}
$$

Finally, taking $\alpha=1$ in the above equality, Lemma 6 is completed.

3.1. A Priori Estimate. Taking $\omega_{j}=u_{t n}$ in (33) and integrating over $(0, t)$, using integration by parts and Lemma 4 , we obtain

$$
\begin{aligned}
\frac{1}{2}\left[\left(1-\int_{0}^{t} h(s) d s\right)\left\|\nabla u_{n}\right\|_{2}^{2}+\frac{2}{\rho+2}\left\|u_{t n}\right\|_{\rho+2}^{\rho+2}+\left(h \circ \nabla u_{n}\right)(t)\right] \\
+\mu_{1} \int_{0}^{t}\left\|u_{t n}\right\|_{2}^{2} d s+\mu_{2} \int_{0}^{t} \int_{\Omega} z_{n}(x, 1, s) u_{t n}(x, s) d x d s \\
\quad+\frac{1}{2} \int_{0}^{t} h(s)\left\|\nabla u_{n}(s)\right\|_{2}^{2} d s-\frac{1}{2} \int_{0}^{t}\left(h^{\prime} \circ \nabla u_{n}\right)(s) d s \\
=\frac{1}{2}\left(\left\|\nabla u_{0}\right\|_{2}^{2}+\frac{2}{\rho+2}\left\|u_{1}\right\|_{\rho+2}^{\rho+2}\right) .
\end{aligned}
$$


Taking $\phi_{j}=z_{n}(\xi / \tau(t))$ in (34) and integrating over $(0, t)$, we get

$$
\begin{aligned}
& \frac{\xi}{2} \int_{\Omega} \int_{0}^{1} z_{n}^{2}(x, \theta, t) d \theta d x \\
& \quad+\xi \int_{0}^{t} \int_{\Omega} \int_{0}^{1} \frac{1-\tau^{\prime}(t) \theta}{\tau(t)} z_{n \theta} z_{n}(x, \theta, s) d \theta d x d s \\
& \quad=\frac{\xi}{2}\left\|z_{0 n}\right\|_{L^{2}(\Omega \times(0,1))^{\circ}}^{2}
\end{aligned}
$$

Now, integrating by parts, we obtain

$$
\begin{aligned}
& \int_{0}^{t} \int_{\Omega} \int_{0}^{1} \frac{1-\tau^{\prime}(t) \theta}{\tau(t)} z_{n \theta} z_{n}(x, \theta, s) d \theta d x d s \\
& =\frac{1}{2} \int_{0}^{t} \int_{\Omega} \int_{0}^{1}\left(\frac{\partial}{\partial \theta} z_{n}^{2}(x, \theta, s) \frac{1-\tau^{\prime}(t) \theta}{\tau(t)}\right) d \theta d x d s \\
& =-\frac{1}{2} \int_{0}^{t} \int_{\Omega} \frac{1-\tau^{\prime}(t) \theta}{\tau(t)} z_{n}^{2}(x, \theta, s) d s d x \\
& \quad+\frac{1}{2} \int_{0}^{t} \int_{\Omega}\left[\frac{\left(1-\tau^{\prime}(t) \theta\right) z_{n}^{2}(x, 1, s)-z_{n}^{2}(x, 0, s)}{\tau(t)}\right] d x d s .
\end{aligned}
$$

It follows from (43) and (44) that

$$
\begin{aligned}
& \frac{\xi}{2} \int_{\Omega} \int_{0}^{1} z_{n}^{2}(x, \theta, t) d \theta d x \\
& \quad+\xi \int_{0}^{t} \int_{\Omega} \int_{0}^{1} \frac{\tau^{\prime}(t) \theta-1}{\tau(t)} z_{n}^{2}(x, \theta, s) d x d s \\
& \quad+\frac{1}{2} \int_{0}^{t} \int_{\Omega}\left[\frac{\left(1-\tau^{\prime}(t) \theta\right) z_{n}^{2}(x, 1, s)-z_{n}^{2}(x, 0, s)}{\tau(t)}\right] d x d s \\
& =\frac{\xi}{2}\left\|z_{0 n}\right\|_{L^{2}(\Omega \times(0,1))}^{2}
\end{aligned}
$$

Summing up (42) and (45), we conclude that

$$
\begin{aligned}
\mathscr{E}_{n}( & t)+\mu_{1} \int_{0}^{t}\left\|u_{t n}\right\|_{2}^{2} d s+\mu_{2} \int_{0}^{t} \int_{\Omega} z_{n}(x, 1, s) u_{t n}(x, s) d x d s \\
& +\frac{1}{2} \int_{0}^{t} h(s)\left\|\nabla u_{n}(s)\right\|_{2}^{2} d s-\frac{1}{2} \int_{0}^{t}\left(h^{\prime} \circ \nabla u_{n}\right)(s) d s \\
& +\frac{\xi}{2} \int_{0}^{t} \int_{\Omega} \frac{\tau^{\prime}(t) \theta-1}{\tau(t)} z_{n}^{2}(x, \theta, s) d x d s \\
& +\frac{1}{2} \int_{0}^{t} \int_{\Omega}\left[\frac{\left(1-\tau^{\prime}(t) \theta\right) z_{n}^{2}(x, 1, s)-z_{n}^{2}(x, 0, s)}{\tau(t)}\right] d x d s \\
= & \frac{1}{2}\left\|\nabla u_{0}\right\|_{2}^{2}+\frac{1}{\rho+2}\left\|u_{t n}\right\|_{\rho+2}^{\rho+2}+\frac{\xi}{2}\left\|z_{0}\right\|_{L^{2}(\Omega \times(0,1))}^{2}=\mathscr{E}_{n}(0),
\end{aligned}
$$

where

$$
\begin{gathered}
\mathscr{E}_{n}(t)=\frac{1}{2}\left[\left(1-\int_{0}^{t} h(s) d s\right)\left\|\nabla u_{n}\right\|_{2}^{2}+\frac{2}{\rho+2}\left\|u_{t n}\right\|_{\rho+2}^{\rho+2}\right. \\
\left.+\left(h \circ \nabla u_{n}\right)(t)\right]+\frac{\xi}{2}\left\|z_{n}\right\|_{L^{2}(\Omega \times(0,1))}^{2} .
\end{gathered}
$$

Using Young's inequality and noticing (20) and (21), we arrive at

$$
\begin{aligned}
& \left(\mu_{1}-\frac{\mu_{2} \xi}{2}\right) \int_{0}^{t}\left\|u_{t n}\right\|_{2}^{2} d s \\
& +\int_{0}^{t} \int_{\Omega}\left[\xi \frac{1-\tau^{\prime}(t)}{2 \tau(t)}-\frac{\mu_{2}}{2 \xi}\right] z_{n}^{2}(x, 1, s) \\
& \quad+\frac{1}{2} \int_{0}^{t} h(s)\left\|\nabla u_{n}(s)\right\|_{2}^{2} d s-\frac{1}{2} \int_{0}^{t}\left(h^{\prime} \circ \nabla u_{n}\right)(s) d s \\
& +\frac{\xi}{2} \int_{0}^{t} \int_{\Omega} \frac{\tau^{\prime}(t) \theta-1}{\tau(t)} z_{n}^{2}(x, \theta, s) d x d s=\mathscr{E}_{n}(0) .
\end{aligned}
$$

Choosing some value of $\tau(t)>0$ and $\theta$ and noticing (20) and (21), we have $\left(\tau^{\prime}(t) \theta-1\right) / \tau(t)>0$. Moreover, choosing some value of $\tau(t)>0$ and $\xi$, we obtain

$$
\mu_{1}-\frac{\mu_{2} \xi}{2}>0, \quad \xi \frac{1-\tau^{\prime}(t)}{2 \tau(t)}-\frac{\mu_{2}}{2 \xi}>0 .
$$

That is,

$$
\sqrt{\frac{\mu_{2} \tau(t)}{1-\tau^{\prime}(t)}}<\xi<\frac{2 \mu_{1}}{\mu_{2}}
$$

In fact, by (20) and (21), we get $\sqrt{\mu_{2} \tau(t)_{0} /(1-d)}<\xi<$ $2 \mu_{1} / \mu_{2}$. From (48) and (50), (G1), and (G1) and Lemma 6, we conclude that we can find a positive $C$ independent of $n$, such that

$$
\mathscr{E}_{n}(t) \leq C
$$

Hence, using the fact that $1-\int_{0}^{t} h(s) d s \geq l$, the estimate (51), and equality (47), we deduce

$$
\begin{gathered}
u_{n} \text { is uniformly bounded in } L^{\infty}\left(0, T ; H_{0}^{1}(\Omega)\right), \\
u_{t n} \text { is uniformly bounded in } L^{\infty}\left(0, T ; L^{2}(\Omega)\right), \\
z_{n} \text { is uniformly bounded in } L^{\infty}\left(0, T ; L^{2}(\Omega \times(0,1))\right) .
\end{gathered}
$$

By (52), we infer that there exist two subsequences $u_{n}, z_{n}$ (still denoted by $u_{n}, z_{n}$ ) and two functions $u$ and $z$, such that

$$
\begin{gathered}
u_{n} \rightarrow u \text { weakly star in } L^{\infty}\left(0, T ; H_{0}^{1}(\Omega)\right), \\
u_{t n} \rightarrow u_{t} \text { weakly star in } L^{\infty}\left(0, T ; L^{2}(\Omega)\right), \\
z_{n} \rightarrow z \text { weakly star in } L^{\infty}\left(0, T ; L^{2}(\Omega \times(0,1))\right) .
\end{gathered}
$$


From (52), we have $u_{n}$ is bounded in $L^{2}\left(0, T ; H_{0}^{1}(\Omega)\right)$ and $u_{t n}$ is bounded in $L^{2}\left(0, T ; L^{2}(\Omega)\right)$. Consequently, $u_{n}$ is bounded in $L^{2}\left(0, T ; L^{2}(\Omega)\right)$. More details are present in [37, pp 1072].

Since the Sobolev embedding $H^{1}\left(0, T ; H^{1}(\Omega)\right) \hookrightarrow$ $L^{2}\left(0, T ; L^{2}(\Omega)\right)$ is compact, using Aubin-Lions theorem (see [45]), we can extract a subsequence of $u_{n}$ (still denoted by $u_{n}$ ), such that

$$
\begin{gathered}
u_{n} \longrightarrow u \text { strongly in } L^{2}\left(0, T ; L^{2}(\Omega)\right), \\
u_{t n} \longrightarrow u_{t} \text { strongly in } L^{2}\left(0, T ; L^{2}(\Omega)\right),
\end{gathered}
$$

which implies $u_{t n} \rightarrow u_{t}$ almost everywhere in $\Omega \times(0, T)$.

Hence,

$\left|u_{t n}\right|^{\rho} u_{t n} \longrightarrow\left|u_{t}\right|^{\rho} u_{t}$ almost everywhere in $\Omega \times(0, T)$.

On the other hand, by the Sobolev embedding theorem and estimate (51), this yields

$$
\begin{aligned}
\left\|\left|u_{t n}\right|^{\rho} u_{t n}\right\|_{L^{2}\left(0, T ; L^{2}(\Omega)\right)} & =\int_{0}^{T} \int_{\Omega}\left|u_{t n}\right|^{2(\rho+1)} d x d t \\
& \leq C_{S}^{2(\rho+1)} \int_{0}^{T}\left\|\nabla u_{t n}\right\|_{2}^{2(\rho+1)} d t \\
& \leq C_{S}^{2(\rho+1)} C^{\rho+1} T,
\end{aligned}
$$

where $C_{S}$ is the Sobolev embedding constant. Thus, using (55), (56), and Lions Lemma [46], we get

$$
\left|u_{t n}\right|^{\rho} u_{t n} \rightarrow\left|u_{t}\right|^{\rho} u_{t} \text { weakly in } L^{2}\left(0, T ; L^{2}(\Omega)\right) .
$$

Let $\mathscr{D}(0, T)$ be the space of $C^{\infty}$ functions with compact support in $(0, T)$. Multiplying the first equation in (33) by $\Theta(t) \in \mathscr{D}(0, T)$ and integrating over $(0, T)$, we conclude that

$$
\begin{aligned}
& -\frac{1}{\rho+1} \int_{0}^{T}\left(\left|u_{t n}\right|^{\rho} u_{t n}, \omega_{j}\right) \Theta_{t}(t) d t \\
& \quad+\int_{0}^{T}\left(\nabla u_{t n}, \nabla \omega_{j}\right) \Theta(t) d t \\
& \quad-\int_{0}^{T} \int_{0}^{t} h(t-s)\left(\nabla u_{t n}, \nabla \omega_{j}\right) \Theta_{t}(t) d s d t \\
& \quad+\int_{0}^{T}\left(\mu_{1} u_{t n}+\mu_{2} z_{n}, \omega_{j}\right) \Theta(t) d t=0 .
\end{aligned}
$$

Noticing that $\left\{\omega_{j}\right\}_{j}^{\infty}$ is a basis of $H_{0}^{1}(\Omega)$, via convergence (53) and (57), we can pass to the limit in (58) and obtain

$$
\begin{aligned}
& \left|u_{t}\right|^{\rho} u_{t t}-\Delta u+\int_{0}^{t} h(t-s) \Delta u(s) d s+\mu_{1} u_{t}(x, t) \\
& +\mu_{2} z(x, 1, t)=0 .
\end{aligned}
$$

Similarly, we get

$$
\tau(t) z_{t}(x, \theta, t)+\left(1-\tau^{\prime}(t) \theta\right) z_{\theta}(x, \theta, t)=0 .
$$

From (53) and given the label of lemma in [46], we obtain

$$
\begin{aligned}
& u_{n}(0) \rightarrow u(0) \text { weakly in } H_{0}^{1}(\Omega) ; \\
& u_{t n}(0) \rightarrow u_{t}(0) \text { weakly in } L^{2}(\Omega) .
\end{aligned}
$$

Therefore, we have $u(0)=u_{0}, u_{t}(0)=u_{1}$. Consequently, the global existence of weak solution is established.

Step 2 (general decay of the energy). First, we introduce the new energy functional $E(t)$ and the perturbed energy $E_{\varepsilon}(t)$; then we apply the perturbed energy method to establish general decay of the energy. More precisely, the method used is based on the construction of suitable Lyapunov functionals $E(t)$ and $E_{\varepsilon}(t)$ satisfying

$$
\frac{d}{d t} E_{\varepsilon}(t) \leq-C_{1} E_{\varepsilon}(t)+C_{2} E(t)^{-r t}
$$

for some positive constants $C_{1}, C_{2}, R$. More details are present in $[3, \mathrm{pp} 1017]$ or $[2,4,16]$.

Now, we introduce the new energy functional as follows:

$$
\begin{aligned}
& E(t) \\
& =E(u, z, t) \\
& =\frac{1}{2}\left[\frac{2}{\rho+2}\left\|u_{t}\right\|_{\rho+2}^{\rho+2}+\left(1-\int_{0}^{t} h(s) d s\right)\|\nabla u\|_{2}^{2}+(h \circ \nabla u)(t)\right] \\
& \quad+\frac{\xi}{2} \int_{t-\tau(t)}^{t} \int_{\Omega} e^{-\lambda(t-s)} u_{t}^{2}(x, s) d s d x,
\end{aligned}
$$

where $\xi, \lambda$ are suitable positive constants.

Next, we will fix $\xi$ such that

$$
\begin{gathered}
2 \mu_{1}-\frac{\left|\mu_{2}\right|}{\sqrt{1-d}}-\xi>0, \quad \xi-\frac{\left|\mu_{2}\right|}{\sqrt{1-d}}>0, \\
\lambda<\frac{1}{\bar{\tau}}\left|\log -\frac{\left|\mu_{2}\right|}{\xi \sqrt{1-d}}\right| .
\end{gathered}
$$

Remark 7. In fact, the existence of such a constant $\xi$ is guaranteed by the assumption (23).

Therefore, we have the following lemma.

Lemma 8. Let (20)-(23) be satisfied and h satisfy (G1). Then, for the solution of problem (1), the energy functional defined by (63) is nonincreasing and satisfies

$$
\begin{aligned}
E^{\prime}(t) \leq & \frac{1}{2}(h \circ \nabla u)(t)-\frac{1}{2} h(t) \int_{\Omega}|\nabla u| d x \\
& -C_{1} \int_{\Omega}\left[u_{t}^{2}(x, t)+u_{t}^{2}(x, t-\tau(t))\right] d x \\
& -\frac{\lambda \xi}{2} \int_{t-\tau(t)}^{t} \int_{\Omega} e^{-\lambda(t-s)} u_{t}^{2}(x, s) d s d x \leq 0,
\end{aligned}
$$

for some positive constant $C_{1}$. 
Proof of Lemma 8. Differentiating (63) and noticing the first equation in (1) together with

$$
(h \circ \nabla u)(t)=\int_{\Omega} \int_{0}^{t} h(t-s)|\nabla u(t)-\nabla u(s)|^{2} d s d x,
$$

we obtain

$$
\begin{aligned}
& E^{\prime}(t)=\int_{\Omega}\left|u_{t}\right|_{\rho+1} u_{t t}-\frac{1}{2} h(t) \int_{\Omega}|\nabla u|^{2} d x \\
& +\left(1-\int_{0}^{t} h(s) d s\right) \int_{\Omega} \nabla u \cdot \nabla u_{t} d x \\
& +\int_{0}^{t} h(t-s) d s \int_{\Omega}[\nabla u(t)-\nabla u(s)] d s d x \\
& +\frac{1}{2} \int_{0}^{t} h^{\prime}(t-s) \int_{\Omega}|\nabla u(t)-\nabla u(s)|^{2} d s d x \\
& +\frac{\xi}{2} \int_{\Omega} u_{t}^{2}(x, t) d x \\
& -\frac{\xi}{2} \int_{\Omega} e^{-\lambda \tau(t)} u_{t}^{2}(x, t-\tau(t))\left(1-\tau^{\prime}(t)\right) d x \\
& -\frac{\lambda \xi}{2} \int_{t-\tau(t)}^{t} \int_{\Omega} e^{-\lambda(t-s)} u_{t}^{2}(x, s) d s d x \\
& =\int_{\Omega} u_{t}\left[\Delta u-\int_{0}^{t} h(t-s) \Delta u(s) d s-\mu_{1} u_{t}(x, t)\right. \\
& \left.-\mu_{2} u_{t}(x, t-\tau(t))\right] d x \\
& -\frac{1}{2} h(t) \int_{\Omega}|\nabla u|^{2} d x \\
& +\int_{\Omega} \nabla u \cdot \nabla u_{t} d x-\int_{0}^{t} h(s) d s \int_{\Omega} \nabla u \cdot \nabla u_{t} d x \\
& +\int_{0}^{t} h(t-s) d s \int_{\Omega}[\nabla u(t)-\nabla u(s)] d s d x \\
& +\frac{1}{2}\left(h^{\prime} \circ \nabla u\right)(t)+\frac{\xi}{2} \int_{\Omega} u_{t}^{2}(x, t) d x \\
& -\frac{\xi}{2} \int_{\Omega} e^{-\lambda \tau(t)} u_{t}^{2}(x, t-\tau(t))\left(1-\tau^{\prime}(t)\right) d x \\
& -\frac{\lambda \xi}{2} \int_{t-\tau(t)}^{t} \int_{\Omega} e^{-\lambda(t-s)} u_{t}^{2}(x, s) d s d x \\
& =-\mu_{1} \int_{\Omega} u_{t}^{2}(x, t) d x-\mu_{2} \int_{\Omega} u_{t}(x, t) u_{t}(x, t-\tau(t)) d x \\
& -\frac{1}{2} h(t) \int_{\Omega}|\nabla u|^{2} d x+\frac{1}{2}\left(h^{\prime} \circ \nabla u\right)(t)
\end{aligned}
$$

$$
\begin{aligned}
& +\frac{\xi}{2} \int_{\Omega} u_{t}^{2}(x, t) d x \\
& -\frac{\xi}{2} \int_{\Omega} e^{-\lambda \tau(t)} u_{t}^{2}(x, t-\tau(t))\left(1-\tau^{\prime}(t)\right) d x \\
& -\frac{\lambda \xi}{2} \int_{t-\tau(t)}^{t} \int_{\Omega} e^{-\lambda(t-s)} u_{t}^{2}(x, s) d s d x
\end{aligned}
$$

Applying Young's inequality, we obtain

$$
\begin{aligned}
& -\mu_{2} \int_{\Omega} u_{t}(x, t) u_{t}(x, t-\tau(t)) d x \\
& \leq \frac{\left|\mu_{2}\right|}{2 \sqrt{1-d}} \int_{\Omega} u_{t}^{2}(x, t) d x \\
& \quad+\frac{\left|\mu_{2}\right| \sqrt{1-d}}{2} \int_{\Omega} u_{t}^{2}(x, t-\tau(t)) d x .
\end{aligned}
$$

Integrating by parts, using the assumption (20), (21) and (67), (68), we arrive at

$$
\begin{aligned}
& E^{\prime}(t) \leq-\mu_{1} \int_{\Omega} u_{t}^{2}(x, t) d x \\
& -\mu_{2} \int_{\Omega} u_{t}(x, t) u_{t}(x, t-\tau(t)) d x \\
& -\frac{1}{2} h(t) \int_{\Omega}|\nabla u|^{2} d x+\frac{1}{2}\left(h^{\prime} \circ \nabla u\right)(t) \\
& +\frac{\xi}{2} \int_{\Omega} u_{t}^{2}(x, t) d x-\frac{\xi}{2}(1-d) e^{-\lambda \bar{\tau}} \\
& \times \int_{\Omega} u_{t}^{2}(x, t-\tau(t)) d x \\
& \left(0<\tau_{0} \leq \tau(t) \leq \bar{\tau}, \tau^{\prime}(t) \leq d<1\right) \\
& -\frac{\lambda \xi}{2} \int_{t-\tau(t)}^{t} \int_{\Omega} e^{-\lambda(t-s)} u_{t}^{2}(x, s) d s d x \\
& \leq \frac{1}{2}\left(h^{\prime} \circ \nabla u\right)(t)-\frac{1}{2} h(t) \int_{\Omega}|\nabla u|^{2} d x \\
& -\left(\mu_{1}-\frac{\left|\mu_{2}\right|}{2 \sqrt{1-d}}-\frac{\xi}{2}\right) \int_{\Omega} u_{t}^{2}(x, t) d x \\
& -\left(\frac{\xi}{2}(1-d) e^{-\lambda \bar{\tau}}-\frac{\left|\mu_{2}\right| \sqrt{1-d}}{2}\right) \\
& \times \int_{\Omega} u_{t}^{2}(x, t-\tau(t)) d x \\
& -\frac{\lambda \xi}{2} \int_{t-\tau(t)}^{t} \int_{\Omega} e^{-\lambda(t-s)} u_{t}^{2}(x, s) d s d x
\end{aligned}
$$

Combining (64) and (69) and the assumptions (G1) and (G2), (65) is established. 
Next, we introduce the following functionals:

$$
\begin{gathered}
\Phi(t)=\frac{1}{\rho+1} \int_{\Omega}\left|u_{t}\right|^{\rho+1} u d x \\
\Psi(t)=-\frac{1}{\rho+1} \int_{\Omega}\left|u_{t}\right|^{\rho+1} \int_{0}^{t} h(t-s)[u(t)-u(s)] d s d x .
\end{gathered}
$$

Set

$$
L(t)=N E(t)+\varepsilon \Phi(t)+\Psi(t)
$$

where $N$ and $\varepsilon$ are suitable positive constants to be determined later.

Remark 9. Indeed, we easily see that, for $\varepsilon$ small enough while $N$ large enough, there exist two positive constants $\alpha_{0}, \alpha_{1}$, such that

$$
\alpha_{0} E(t) \leq L(t) \leq \alpha_{1} E(t), \quad \forall t \geq 0
$$

Concerning the estimates of $\Phi(t), \Psi(t)$, we have the following lemmas.

Lemma 10. Under the assumption (G1), the functional $\Phi(t)$ satisfies the estimate

$$
\begin{aligned}
\Phi^{\prime}(t) & \\
\leq & -\frac{l}{2} \int_{\Omega}|\nabla u|^{2} d x \\
& +C_{2} \int_{\Omega}\left[u_{t}^{2}(x, t)+u_{t}^{2}(x, t-\tau(t))\right] d x+C_{3}(h \circ \nabla u) .
\end{aligned}
$$

Proof of Lemma 10. Differentiating (70) and integrating by parts, we get

$$
\begin{aligned}
\Phi^{\prime}(t)= & \int_{\Omega}\left|u_{t}\right|^{\rho+1} u_{t t} u d x+\frac{1}{\rho+1} \int_{\Omega}\left|u_{t}\right|^{\rho+2} d x \\
= & \int_{\Omega} u\left[\Delta u-\int_{0}^{t} h(t-s) \Delta u(s) d s-\mu_{1} u_{t}(x, t)\right. \\
& \left.-\mu_{2} u_{t}(x, t-\tau(t))\right] d x \\
& +\frac{1}{\rho+1} \int_{\Omega}\left|u_{t}\right|^{\rho+2} d x \\
= & -\int_{\Omega}|\nabla u|^{2} d x+\int_{\Omega} \nabla u \cdot \int_{0}^{t} h(t-s) \nabla u(s) d s d x \\
& -\mu_{1} \int_{\Omega} u u_{t}(x, t) d x \\
& -\mu_{2} \int_{\Omega} u u_{t}(x, t-\tau(t)) d x \frac{1}{\rho+1} \int_{\Omega}\left|u_{t}\right|^{\rho+2} d x
\end{aligned}
$$

$$
\begin{aligned}
= & -l \int_{\Omega}|\nabla u|^{2} d x \\
& +\int_{\Omega} \nabla u \cdot \int_{0}^{t} h(t-s)[\nabla u(s)-\nabla u(t)] d s d x \\
& -\mu_{1} \int_{\Omega} u u_{t}(x, t) d x-\mu_{2} \int_{\Omega} u u_{t}(x, t-\tau(t)) d x \\
& +\frac{1}{\rho+1} \int_{\Omega}\left|u_{t}\right|^{\rho+2} d x .
\end{aligned}
$$

Using Young's inequality and (G1), we obtain (see [2])

$$
\begin{aligned}
\int_{\Omega} \nabla u \cdot \int_{0}^{t} h(t-s)[\nabla u(s)-\nabla u(t)] d s d x \\
\leq \delta \int_{\Omega}|\nabla u|^{2} d x \\
\quad+\frac{1}{4 \delta} \int_{\Omega}\left[\int_{0}^{t} h(t-s)|\nabla u(s)-\nabla u(t)| d s\right]^{2} d x \\
\leq \delta \int_{\Omega}|\nabla u|^{2} d x+\frac{1-l}{4}(h \circ \nabla u)(t), \quad \forall \delta>0 .
\end{aligned}
$$

Also, applying Young's and Poincaré's inequality yields

$$
\begin{gathered}
-\mu_{1} \int_{\Omega} u u_{t}(x, t) d x \leq \delta \int_{\Omega}|\nabla u|^{2} d x+C(\delta) \int_{\Omega} u_{t}^{2}(x, t) d x, \\
-\mu_{2} \int_{\Omega} u u_{t}(x, t-\tau(t)) d x \\
\leq \delta \int_{\Omega}|\nabla u|^{2} d x+C(\delta) \int_{\Omega} u_{t}^{2}(x, t-\tau(t)) d x .
\end{gathered}
$$

Noticing (75)-(77) and choosing $\delta$ small enough, we obtain estimate (74).

Lemma 11. Under the assumption (G1), the functional $\Psi(t)$ satisfies the estimate

$$
\begin{aligned}
\Psi^{\prime}(t) \leq & -\left(\int_{0}^{t} h(s) d s-2 \delta\right) \int_{\Omega} u_{t}^{2} d x+\delta \int_{\Omega}|\nabla u|^{2} d x \\
& +\frac{C_{4}}{\delta}(h \circ \nabla u)(t)-\frac{C_{5}}{\delta}\left(h^{\prime} \circ \nabla u\right)(t) \\
& +\delta \int_{\Omega} u_{t}^{2}(x, t-\tau(t)) d x .
\end{aligned}
$$


Proof of Lemma 11. Differentiating (71), integrating by parts, and noticing the first equation in (1), we have

$$
\begin{aligned}
& \Psi^{\prime}(t)=-\int_{\Omega}\left|u_{t}\right|^{\rho} u_{t t} \int_{0}^{t} h(t-s)[u(t)-u(s)] d s d x \\
& -\frac{1}{\rho+1} \int_{\Omega}\left|u_{t}\right|^{\rho} u_{t} \int_{0}^{t} h^{\prime}(t-s)[u(t)-u(s)] d s d x \\
& -\left(\int_{0}^{t} h(s) d s\right) \int_{\Omega} \frac{1}{\rho+1}\left|u_{t}\right|^{\rho+2} d x \\
& =\int_{\Omega}\left[-\Delta u+\int_{0}^{t} h(t-s) \Delta u(s) d s\right. \\
& \left.+\mu_{1} u_{t}(x, t)+\mu_{2} u_{t}(x, t-\tau(t))\right] \\
& \times \int_{0}^{t} h(t-s)[u(t)-u(s)] d s d x \\
& -\frac{1}{\rho+1} \int_{\Omega}\left|u_{t}\right|^{\rho} u_{t} \int_{0}^{t} h^{\prime}(t-s)[u(t)-u(s)] d s d x \\
& -\left(\int_{0}^{t} h(s) d s\right) \int_{\Omega} \frac{1}{\rho+1}\left|u_{t}\right|^{\rho+2} d x \\
& =\int_{\Omega} \nabla u \cdot \int_{0}^{t} h(t-s)[\nabla u(t)-\nabla u(s)] d s d x \\
& +\int_{\Omega} \int_{0}^{t} h(t-s) \Delta u(s) d s \\
& \times \int_{0}^{t} h(t-s)[u(t)-u(s)] d s d x \\
& +\int_{\Omega}\left[\int_{0}^{t} h(t-s)[u(t)-u(s)] d s\right] \\
& \times\left[\mu_{1} u_{t}(x, t)+\mu_{2} u_{t}(x, t-\tau(t))\right] d x \\
& -\frac{1}{\rho+1} \int_{\Omega}\left|u_{t}\right|^{\rho} u_{t} \int_{0}^{t} h^{\prime}(t-s)[u(t)-u(s)] d s d x \\
& -\left(\int_{0}^{t} h(s) d s\right) \int_{\Omega} \frac{1}{\rho+1}\left|u_{t}\right|^{\rho+2} d x .
\end{aligned}
$$

Observe that

$$
\begin{gathered}
\int_{\Omega} \int_{0}^{t} h(t-s) \Delta u(s) d s \int_{0}^{t} h(t-s)[u(t)-u(s)] d s d x \\
=-\int_{\Omega}\left[\int_{0}^{t} h(t-s) \nabla u(s) d s\right. \\
\left.\quad \times \int_{0}^{t} h(t-s)[\nabla u(t)-\nabla u(s)] d s\right] d x
\end{gathered}
$$

$$
\begin{aligned}
=- & \int_{\Omega}\left[\int_{0}^{t} h(t-s)[\nabla u(s)-\nabla u(t)+\nabla u(s)] d s\right. \\
& \left.\times \int_{0}^{t} h(t-s)[\nabla u(t)-\nabla u(s)] d s\right] d x \\
=- & \int_{\Omega}\left[\int_{0}^{t} h(t-s)[\nabla u(s)-\nabla u(t)] d s\right]^{2} d x \\
& -\left(\int_{0}^{t} h(t) d s\right) \\
& \times \int_{\Omega} \nabla u \cdot \int_{0}^{t} h(t-s)[\nabla u(t)-\nabla u(s)] d s d x .
\end{aligned}
$$

It follows from (79) and (80) that

$$
\begin{aligned}
\Psi^{\prime}(t)= & \left(1-\int_{0}^{t} h(s) d s\right) \\
& \times \int_{\Omega} \nabla u \cdot \int_{0}^{t} h(t-s)[\nabla u(t)-\nabla u(s)] d s d x \\
& +\int_{\Omega}\left[\int_{0}^{t} h(t-s)[\nabla u(s)-\nabla u(t)] d s\right]^{2} d x \\
& +\int_{\Omega}\left[\int_{0}^{t} h(t-s)[u(t)-u(s)] d s\right] \\
& +\frac{1}{\rho+1} \int_{\Omega} \mid \mu_{1} u_{t}(x, t)+\mu_{2} u_{t} \int_{0}^{t} h^{\prime}(t-s)[u(t)-u(s)] d s d x \\
& -\left(\int_{0}^{t} h(s) d s\right) \int_{\Omega} \frac{1}{\rho+1}\left|u_{t}\right|^{\rho+2} d x .
\end{aligned}
$$

Using Young's and Poincaré's inequality, we get (see [2])

$$
\begin{aligned}
& \left(1-\int_{0}^{t} h(s) d s\right) \int_{\Omega} \nabla u \cdot \int_{0}^{t} h(t-s)[\nabla u(t)-\nabla u(s)] d s d x \\
& \quad \leq \delta \int_{\Omega}|\nabla u|^{2} d x+\frac{C}{\delta}(h \circ \nabla u)(t), \\
& -\frac{1}{\rho+1} \int_{\Omega}\left|u_{t}\right|^{\rho} u_{t} \int_{0}^{t} h^{\prime}(t-s)[u(t)-u(s)] d s d x \\
& \quad \leq \delta \int_{\Omega} u_{t}^{2} d x-\frac{C}{\delta}\left(h^{\prime} \circ \nabla u\right)(t) .
\end{aligned}
$$

From (81) and (82), we derive Lemma 11.

Now, we are ready to finalize our proof of general decay of the energy. Since $h$ is positive, we have

$$
\int_{0}^{t} h(s) d s \geq \int_{0}^{t_{0}} h(s) d s=g_{0}, \quad \forall t \geq t_{0} .
$$


It follows from (65), (72), (74), and (78) that

$$
\begin{aligned}
& L^{\prime}(t)=N E(t)+\varepsilon \Phi^{\prime}(t)+\Psi^{\prime}(t) \\
& \leq \frac{N}{2}\left(h^{\prime} \circ \nabla u\right)(t)-\frac{N}{2} h(t) \int_{\Omega}|\nabla u|^{2} d x \\
& -N C_{1} \int_{\Omega}\left[u_{t}^{2}(x, t)+u_{t}^{2}(x, t-\tau(t))\right] d x \\
& -\frac{\lambda \xi N}{2} \int_{t-\tau(t)}^{t} \int_{\Omega} e^{-\lambda(t-s)} u_{t}^{2}(x, s) d s d x \\
& +\varepsilon C_{2} \int_{\Omega}\left[u_{t}^{2}(x, t)+u_{t}^{2}(x, t-\tau(t))\right] d x \\
& -\frac{\varepsilon l}{2} \int_{\Omega}|\nabla u|^{2} d x P+\varepsilon C_{3}(h \circ \nabla u)(t) \\
& -\left(\int_{0}^{t} h(s) d s-2 \delta\right) \int_{\Omega} u_{t}^{2} d x+\delta \int_{\Omega}|\nabla u|^{2} d x \\
& +\frac{C_{4}}{\delta}(h \circ \nabla u)(t)-\frac{C_{5}}{\delta}\left(h^{\prime} \circ \nabla u\right)(t) \\
& +\delta \int_{\Omega} u_{t}^{2}(x, t-\tau(t)) d x \\
& =-\left[\left(N C_{1}+g_{0}\right)-2 \delta-\varepsilon C_{2}\right] \int_{\Omega} u_{t}^{2}(x, t) d x \\
& +\left(\varepsilon C_{3}+\frac{C_{4}}{\delta}\right)(h \circ \nabla u)(t) \\
& +\left(\frac{N}{2}-\frac{C_{5}}{\delta}\right)\left(h^{\prime} \circ \nabla u\right)(t) \\
& -\left(\frac{\varepsilon l}{2}-\delta\right) \int_{\Omega}|\nabla u|^{2} d x \\
& -\left(N C_{1}-\delta-\varepsilon C_{2}\right) \int_{\Omega} u_{t}^{2}(x, t-\tau(t)) \\
& -\frac{\lambda \xi N}{2} \int_{t-\tau(t)}^{t} \int_{\Omega} e^{-\lambda(t-s)} u_{t}^{2}(x, s) d s d x .
\end{aligned}
$$

If we choose some constants in the inequality (84), such that

$$
\begin{gathered}
a_{1}=\left(N C_{1}+g_{0}\right)-2 \delta-\varepsilon C_{2}>0, \\
a_{3}=N C_{1}-\delta-\varepsilon C_{2}>0, \quad a_{2}=\frac{\varepsilon l}{2}-\delta>0, \\
a_{4}=\frac{N}{2}-\frac{C_{5}}{\delta}>0, \quad a_{5}=\varepsilon C_{3}+\frac{C_{4}}{\delta}>0, \\
a_{6}=\frac{\lambda \xi N}{2},
\end{gathered}
$$

then we conclude that

$$
\begin{aligned}
L^{\prime}(t) \leq & -a_{1} \int_{\Omega} u_{t}^{2}(x, t) d x-a_{2} \int_{\Omega}|\nabla u|^{2} d x \\
& +a_{4}\left(h^{\prime} \circ \nabla u\right)(t)+a_{5}(h \circ \nabla u)(t) \\
& -a_{6} \int_{t-\tau(t)}^{t} \int_{\Omega} e^{-\lambda(t-s)} u_{t}^{2}(x, s) d s d x .
\end{aligned}
$$

Hence, we have two cases to consider the general decay results as follows.

Case $1(p=1)$. Choosing some values of $a_{1}, a_{2}, a_{3}, a_{4}, a_{5}, a_{6}$ and noticing the definition of $E(t)$ (see (63)), we conclude that there exists a constant $\beta_{1}>0$, such that

$$
L^{\prime}(t) \leq-\beta_{1} E(t), \quad \forall t \geq 0 .
$$

Therefore, by Remark 9 and (87), we get

$$
L^{\prime}(t) \leq-\frac{\beta_{1}}{\alpha_{1}} E(t), \quad \forall t \geq 0 .
$$

Integrating $(88)$ over $(0, t)$, we obtain

$$
L(t) \leq L(0) e^{-\left(\beta_{1} / \alpha_{1}\right) t}, \quad \forall t \geq 0 .
$$

Observing Remark 9 (i.e., $\left.\alpha_{0} E(t) \leq L(t) \leq \alpha_{1} E(t)\right)$ and (89), we derive

$$
\alpha_{0} E(t) \leq L(t) \leq L(0) e^{-\left(\beta_{1} / \alpha_{1}\right) t}, \quad \forall t \geq 0 .
$$

That is,

$$
E(t) \leq \frac{L(0)}{\alpha_{0}} e^{-\left(\beta_{1} / \alpha_{1}\right) t} \doteq K e^{k t}, \quad p=1, \forall t \geq 0 .
$$

Assuming $K=L(0) / \alpha_{0}, k=\beta_{1} / \alpha_{1}$, we obtain the exponential decay of the energy. So, (26) is established.

Case $2(1<p<3 / 2)$. Due to (G2), we easily see that

$$
\int_{0}^{\infty} h^{1-r}(s) d s<\infty, \quad 0 \leq r \leq 2-p .
$$

From the sketch of proof of Lemma 6, we observe that

$$
\begin{aligned}
(h \circ \nabla u)(t) \leq & C\left[\int_{0}^{\infty} h^{1-\alpha}(s) d s E(0)\right]^{(p-1+\alpha) /(p-1)} \\
& \times\left[\left(h^{p} \circ \nabla u\right)(t)\right]^{\alpha /(p-1+\alpha)} .
\end{aligned}
$$

Thus, for $\sigma>1$, using (63) and (93), we get

$$
\begin{aligned}
E^{\sigma}(t) \leq & C\left[E^{\sigma-1}(0)\left(\left\|u_{t}\right\|_{\rho+2}^{\rho+2}+\|\nabla u\|_{2}^{2}+\left\|u_{t}\right\|_{2}^{2}\right)\right. \\
& \left.+(h \circ \nabla u)^{\sigma}(t)\right] \\
\leq & C E^{\sigma-1}(0)\left[\left\|u_{t}\right\|_{\rho+2}^{\rho+2}+\|\nabla u\|_{2}^{2}+\left\|u_{t}\right\|_{2}^{2}\right] \\
+ & C\left[\left(\int_{0}^{\infty} h^{1-\alpha}(s) d s\right) E(0)\right]^{(p-1+\alpha) /(p-1)} \\
& \times\left[\left(h^{p} \circ \nabla u\right)(t)\right]^{\sigma \alpha /(p-1+\alpha)} .
\end{aligned}
$$


Choosing $\alpha=1 / 2, \sigma=2 p-1$ (i.e., $\sigma \alpha /(p-1+\alpha)=1$ ) (94) reduces to

$$
E^{\sigma}(t) \leq C\left[\left\|u_{t}\right\|_{\rho+2}^{\rho+2}+\|\nabla u\|_{2}^{2}+\left\|u_{t}\right\|_{2}^{2}+\left(h^{p} \circ \nabla u\right)(t)\right] .
$$

Combining (86) and (87) with Remark 9, we obtain

$$
L^{\prime}(t) \leq-\frac{\beta_{1}}{C} \alpha_{1}^{\sigma} L^{\sigma}(t), \quad \forall t \geq 0
$$

A simple integration of $(96)$ over $(0, t)$ yields

$$
L^{\prime}(t) \leq C_{6}(1+t)^{-1 /(\sigma-1)}, \quad \forall t \geq 0 .
$$

As a consequence of (97), we obtain

$$
\int_{0}^{\infty} L(t) d t+\sup _{t \geq 0} t F(t)<\infty .
$$

So, by using Lemma 6, we have

$$
\begin{aligned}
(h \circ \nabla u)(t) & \\
\leq & C\left[\int_{0}^{t}\|u(s)\|_{H^{1}(s)} d s+t\|u\|_{H^{1}(\Omega)}\right]^{(p-1) / p} \\
& \times\left(h^{p} \circ \nabla u\right)^{1 / p}(t) \\
\leq & C\left[\int_{0}^{t} F(s) d s+t F(t)\right]^{(p-1) / p}\left(h^{p} \circ \nabla u\right)^{1 / p}(t) \\
\leq & C\left(h^{p} \circ \nabla u\right)^{1 / p}(t)
\end{aligned}
$$

which implies that

$$
\left(h^{p} \circ \nabla u\right)(t) \geq C(h \circ \nabla u)^{p}(t) .
$$

Consequently, from (86) and (100), we have

$$
L^{\prime}(t) \leq-C_{7}\left[\left\|u_{t}\right\|_{\rho+2}^{\rho+2}+\|\nabla u\|_{2}^{2}+\left\|u_{t}\right\|_{2}^{2}+(h \circ \nabla u)^{p}(t)\right],
$$$$
\forall t \geq 0 \text {. }
$$

On the other hand, similarly to (95), we

$$
\begin{array}{r}
E^{p}(t) \leq C_{8}\left[\left\|u_{t}\right\|_{\rho+2}^{\rho+2}+\|\nabla u\|_{2}^{2}+\left\|u_{t}\right\|_{2}^{2}+(h \circ \nabla u)^{p}(t)\right], \\
\forall t \geq 0 .
\end{array}
$$

Then, it follows from Remark 9, (101), and (102) that

$$
L^{\prime}(t) \leq-C_{9} L^{p}(t), \quad \forall t \geq 0 .
$$

A simple integration of $(103)$ over $(0, t)$ gives

$$
L^{\prime}(t) \leq K(1+t)^{-1 /(p-1)}, \quad \forall t \geq 0 .
$$

By (104) and Remark 9, we obtain the polynomial decay of the energy. That is,

$$
E(t) \leq K(1+t)^{-1 /(p-1)}, \quad \forall t \geq 0 .
$$

Thus, our main result is completed.
Remark 12. Our novel contribution is to show that our work improves earlier result in [37] in which only the exponential decay was investigated. More precisely, Kirane and SaidHouari [37] considered the exponential decay of problem (1) with a constant delay (i.e., $\tau(t)=\tau$ ) and velocity-independent material density (i.e., $\rho=0$ ).

Remark 13. By using the fact that energy $E$ is bounded on $\left[0, t_{0}\right]$, we can easily show that estimates (26) and (27) hold for $t \geq 0$. (See, for instance, [2].)

\section{Further Remarks}

In this section, we address some interesting problems of nonlinear viscoelastic equation with time-varying delay effects and velocity-dependent material density. Here, we mention some of them.

(1) An interesting problem is to show the well-posedness and stabilization of the nonlinear viscoelastic equation with boundary feedback with respect to timevarying delay effects. What will happen if the controller with time-varying delay effects is in the equation instead of on the boundary? More precisely, in our forthcoming work, we will investigate the wellposedness and general decay properties of the solutions for the following nonlinear viscoelastic equation with velocity-dependent material density:

$$
\begin{gathered}
\left|u_{t}\right|^{\rho} u_{t t}-\Delta u+\int_{0}^{t} h(t-s) \Delta u(s) d s=0, \quad \text { in } \Omega \times[0, \infty), \\
u(x, t)=0, \quad \text { on } \Gamma_{0} \times(0, \infty),
\end{gathered}
$$

$\frac{\partial u}{\partial \nu}+\mu_{1} u_{t}(x, t)+\mu_{2} u_{t}(x, t-\tau(t))=0, \quad$ on $\Gamma_{1} \times[0, \infty)$,

$$
\begin{gathered}
u(x, 0)=u_{0}(x), \quad u_{t}(x, 0)=u_{1}(x), \quad \text { in } \Omega, \\
u_{t}(x, t-\tau(t))=f(x, t), \quad \text { on } \Gamma_{1} \times(-\tau(0), 0),
\end{gathered}
$$

where $\Omega$ is bounded domain of $R^{n}$ and $n \geq 1$ with a smooth boundary $\Gamma$ and let $\Gamma_{0}, \Gamma_{1}$ be a partition of $\Gamma$ such that $\overline{\Gamma_{0}} \cap \overline{\Gamma_{1}}=\emptyset, \Gamma_{0} \neq \emptyset, \Gamma_{1} \neq \emptyset, v=\left(v_{1}, v_{2} \cdots v_{n}\right)$ denotes the unit outward normal to $\Gamma$.

(2) Another interesting problem is to give a positive answer of the open problem given by Kirane and Said-Houari [37]. That is, the linear damping term $\mu_{1} u_{t}$ in the first equation of (16) plays a decisive role in their proofs. Thus, the problem of whether the stability properties they have proved are preserved when $\mu_{1}=0$ is open. In order to overcome the above difficulty, our main idea is to contrast the effects of the time-varying delay by using the dissipative nonlinear 
boundary feedback. That is, in our future work, we investigate the following problem:

$$
\begin{gathered}
\left|u_{t}\right|^{\rho} u_{t t}-\Delta u+\int_{0}^{t} h(t-s) \Delta u(s) d s \\
+\mu_{2} u_{t}(x, t-\tau(t))=0, \quad \text { in } \Omega \times[0, \infty), \\
u(x, t)=0, \quad \text { on } \Gamma_{0} \times(0, \infty), \\
\frac{\partial u}{\partial \nu}+g\left(u_{t}(x, t)\right)=0, \quad \text { on } \Gamma_{1} \times[0, \infty), \\
u(x, 0)=u_{0}(x), \quad u_{t}(x, 0)=u_{1}(x), \quad \text { in } \Omega, \\
u_{t}(x, t-\tau(t))=f(x, t), \quad \text { on } \Gamma_{1} \times(-\tau(0), 0),
\end{gathered}
$$

where $\mu_{2}$ is constant and $g\left(u_{t}\right)$ is the dissipative nonlinear boundary feedback.

\section{Conflict of Interests}

The authors declare that there is no conflict of interests regarding the publication of this paper.

\section{Acknowledgments}

The authors are highly grateful for the anonymous referee's valuable comments and suggestions on this paper. The authors would like to express their sincere gratitude to Dr. Professor Jianhua Huang and Dr. Professor Zhenhai Liu for their enthusiastic guidance and constant encouragement. Also, the authors would like to thank Dr. Professor Ming Bao Sun for always helping and being concerned. This work was supported by China Postdoctoral Science Foundation no. 2013M532169, NSF of Hunan Province Grant no. 11JJ3004, and NNSF of China Grant nos. 11371367, 11271087, 11211120146, and 11271118 .

\section{References}

[1] K. Ammari, S. Nicaise, and C. Pignotti, "Feedback boundary stabilization of wave equations with interior delay," Systems \& Control Letters, vol. 59, no. 10, pp. 623-628, 2010.

[2] Z.-Y. Zhang, Z.-H. Liu, and X.-Y. Gan, "Global existence and general decay for a nonlinear viscoelastic equation with nonlinear localized damping and velocity-dependent material density," Applicable Analysis, vol. 92, no. 10, pp. 2021-2048, 2013.

[3] Z.-Y. Zhang and X.-J. Miao, "Global existence and uniform decay for wave equation with dissipative term and boundary damping," Computers \& Mathematics with Applications, vol. 59, no. 2, pp. 1003-1018, 2010.

[4] Z.-Y. Zhang, Z.-H. Liu, X.-J. Miao, and Y.-Z. Chen, "Global existence and uniform stabilization of a generalized dissipative Klein-Gordon equation type with boundary damping," Journal of Mathematical Physics, vol. 52, no. 2, Article ID 023502, 2011.

[5] Z.-Y. Zhang, Z.-H. Liu, and X.-J. Miao, "Estimate on the dimension of global attractor for nonlinear dissipative Kirchhoff equation," Acta Applicandae Mathematicae, vol. 110, no. 1, pp. 271-282, 2010.
[6] M. M. Cavalcanti, V. N. Domingos Cavalcanti, R. Fukuoka, and J. A. Soriano, "Asymptotic stability of the wave equation on compact surfaces and locally distributed damping - a sharp result," Transactions of the American Mathematical Society, vol. 361, no. 9, pp. 4561-4580, 2009.

[7] M. M. Cavalcanti, V. N. Domingos Cavalcanti, R. Fukuoka, and J. A. Soriano, "Asymptotic stability of the wave equation on compact manifolds and locally distributed damping: a sharp result," Archive for Rational Mechanics and Analysis, vol. 197, no. 3, pp. 925-964, 2010.

[8] M. M. Cavalcanti, V. N. Domingos Cavalcanti, and J. A. Soriano, "Exponential decay for the solution of semilinear viscoelastic wave equations with localized damping," Electronic Journal of Differential Equations, vol. 44, pp. 1-14, 2002.

[9] S. Berrimi and S. A. Messaoudi, "Exponential decay of solutions to a viscoelastic equation with nonlinear localized damping," Electronic Journal of Differential Equations, vol. 88, pp. 1-10, 2004.

[10] M. M. Cavalcanti and H. P. Oquendo, "Frictional versus viscoelastic damping in a semilinear wave equation," SIAM Journal on Control and Optimization, vol. 42, no. 4, pp. 1310-1324, 2003.

[11] Z.-Y. Zhang, Z.-H. Liu, X.-J. Miao, and Y.-Z. Chen, "A note on decay properties for the solutions of a class of partial differential equation with memory," Journal of Applied Mathematics and Computing, vol. 37, no. 1-2, pp. 85-102, 2011.

[12] W. Liu, "General decay rate estimate for a viscoelastic equation with weakly nonlinear time-dependent dissipation and source terms," Journal of Mathematical Physics, vol. 50, no. 11, Article ID 113506, 2009.

[13] W. Liu, "Uniform decay of solutions for a quasilinear system of viscoelastic equations," Nonlinear Analysis. Theory, Methods \& Applications, vol. 71, no. 5-6, pp. 2257-2267, 2009.

[14] W. Liu, "General decay and blow-up of solution for a quasilinear viscoelastic problem with nonlinear source," Nonlinear Analysis. Theory, Methods \& Applications, vol. 73, no. 6, pp. 1890-1904, 2010.

[15] W. Liu, "General decay of solutions to a viscoelastic wave equation with nonlinear localized damping," Annales Academice Scientiarum Fennice. Mathematica, vol. 34, no. 1, pp. 291-302, 2009.

[16] W. Liu, "General decay of solutions of a nonlinear system of viscoelastic equations," Acta Applicandae Mathematicae, vol. 110, no. 1, pp. 153-165, 2010.

[17] W. Liu, "Exponential or polynomial decay of solutions to a viscoelastic equation with nonlinear localized damping," Journal of Applied Mathematics and Computing, vol. 32, no. 1, pp. 59-68, 2010.

[18] X. Han and M. Wang, "Global existence and uniform decay for a nonlinear viscoelastic equation with damping," Nonlinear Analysis. Theory, Methods \& Applications, vol. 70, no. 9, pp. 3090-3098, 2009.

[19] X. Han and M. Wang, "General decay of energy for a viscoelastic equation with nonlinear damping," Journal of the Franklin Institute, vol. 347, no. 5, pp. 806-817, 2010.

[20] X. Han and M. Wang, "Global existence and asymptotic behavior for a coupled hyperbolic system with localized damping," Nonlinear Analysis. Theory, Methods \& Applications, vol. 72, no. 2, pp. 965-986, 2010.

[21] X. Han and M. Wang, "Energy decay rate for a coupled hyperbolic system with nonlinear damping," Nonlinear Analysis. Theory, Methods \& Applications, vol. 70, no. 9, pp. 3264-3272, 2009. 
[22] X. Han and M. Wang, "General decay of energy for a viscoelastic equation with nonlinear damping," Mathematical Methods in the Applied Sciences, vol. 32, no. 3, pp. 346-358, 2009.

[23] M. M. Cavalcanti, V. N. Domingos Cavalcanti, J. S. Prates Filho, and J. A. Soriano, "Existence and uniform decay rates for viscoelastic problems with nonlinear boundary damping," Differential and Integral Equations, vol. 14, no. 1, pp. 85-116, 2001.

[24] S. A. Messaoudi and N.-E. Tatar, "Exponential and polynomial decay for a quasilinear viscoelastic equation," Nonlinear Analysis. Theory, Methods \& Applications, vol. 68, no. 4, pp. 785-793, 2008.

[25] N.-E. Tatar, "Exponential decay for a viscoelastic problem with a singular kernel," Zeitschrift für Angewandte Mathematik und Physik, vol. 60, no. 4, pp. 640-650, 2009.

[26] S. Berrimi and S. A. Messaoudi, "Existence and decay of solutions of a viscoelastic equation with a nonlinear source," Nonlinear Analysis. Theory, Methods \& Applications, vol. 64, no. 10, pp. 2314-2331, 2006.

[27] S. A. Messaoudi, "General decay of the solution energy in a viscoelastic equation with a nonlinear source," Nonlinear Analysis. Theory, Methods \& Applications, vol. 69, no. 8, pp. 2589-2598, 2008.

[28] W. Liu, "Asymptotic behavior of solutions of time-delayed Burgers' equation," Discrete and Continuous Dynamical Systems $B$, vol. 2, no. 1, pp. 47-56, 2002.

[29] Y. F. Shang, G. Q. Xu, and Y. L. Chen, "Stability analysis of EulerBernoulli beam with input delay in the boundary control," Asian Journal of Control, vol. 14, no. 1, pp. 186-196, 2012.

[30] R. Datko, "Not all feedback stabilized hyperbolic systems are robust with respect to small time delays in their feedbacks," SIAM Journal on Control and Optimization, vol. 26, no. 3, pp. 697-713, 1988.

[31] R. Datko, J. Lagnese, and M. P. Polis, "An example on the effect of time delays in boundary feedback stabilization of wave equations," SIAM Journal on Control and Optimization, vol. 24, no. 1, pp. 152-156, 1986.

[32] S. Nicaise and C. Pignotti, "Stability and instability results of the wave equation with a delay term in the boundary or internal feedbacks," SIAM Journal on Control and Optimization, vol. 45, no. 5, pp. 1561-1585, 2006.

[33] S. Nicaise and C. Pignotti, "Stabilization of the wave equation with boundary or internal distributed delay," Differential and Integral Equations, vol. 21, no. 9-10, pp. 935-958, 2008.

[34] G. Q. Xu, S. P. Yung, and L. K. Li, "Stabilization of wave systems with input delay in the boundary control," ESAIM. Control, Optimisation and Calculus of Variations, vol. 12, no. 4, pp. 770785, 2006.

[35] E. M. Ait Benhassi, K. Ammari, S. Boulite, and L. Maniar, "Feedback stabilization of a class of evolution equations with delay," Journal of Evolution Equations, vol. 9, no. 1, pp. 103-121, 2009.

[36] S. Nicaise, C. Pignotti, and J. Valein, "Exponential stability of the wave equation with boundary time-varying delay," Discrete and Continuous Dynamical Systems S, vol. 4, no. 3, pp. 693-722, 2011.

[37] M. Kirane and B. Said-Houari, "Existence and asymptotic stability of a viscoelastic wave equation with a delay," Zeitschrift für Angewandte Mathematik und Physik, vol. 62, no. 6, pp. 10651082, 2011.
[38] T. Caraballo, J. Real, and L. Shaikhet, "Method of Lyapunov functionals construction in stability of delay evolution equations," Journal of Mathematical Analysis and Applications, vol. 334, no. 2, pp. 1130-1145, 2007.

[39] Z.-Y. Zhang, Z.-H. Liu, X.-J. Miao, and Y.-Z. Chen, "Stability analysis of heat flow with boundary time-varying delay effect," Nonlinear Analysis. Theory, Methods \& Applications, vol. 73, no. 6, pp. 1878-1889, 2010.

[40] S. Nicaise and C. Pignotti, "Interior feedback stabilization of wave equations with time dependent delay," Electronic Journal of Differential Equations, vol. 41, pp. 1-20, 2011.

[41] S. Nicaise, J. Valein, and E. Fridman, "Stability of the heat and of the wave equations with boundary time-varying delays," Discrete and Continuous Dynamical Systems S, vol. 2, no. 3, pp. 559-581, 2009.

[42] S. Nicaise and J. Valein, "Stabilization of the wave equation on 1D networks with a delay term in the nodal feedbacks," Networks and Heterogeneous Media, vol. 2, no. 3, pp. 425-479, 2007.

[43] E. Fridman, S. Nicaise, and J. Valein, "Stabilization of second order evolution equations with unbounded feedback with timedependent delay," SIAM Journal on Control and Optimization, vol. 48, no. 8, pp. 5028-5052, 2010.

[44] S. Nicaise and J. Valein, "Stabilization of second order evolution equations with unbounded feedback with delay," ESAIM. Control, Optimisation and Calculus of Variations, vol. 16, no. 2, pp. 420-456, 2010.

[45] J.-L. Lions, Quelques Méthodes de Résolution des Problèmes aux Limites Non Linéaires, Dunod, Gauthier-Villars, Paris, France, 1969.

[46] S. Zheng, Nonlinear Evolution Equations, CRC Press, Boca Raton, Fla, USA, 2004. 


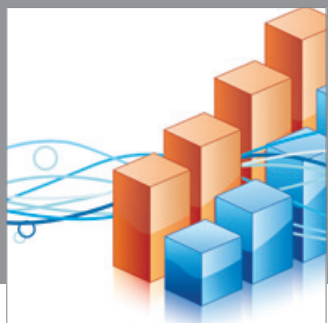

Advances in

Operations Research

mansans

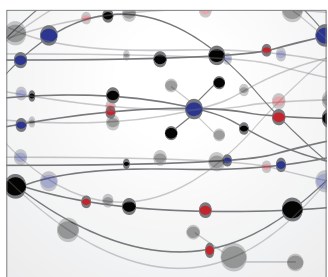

The Scientific World Journal
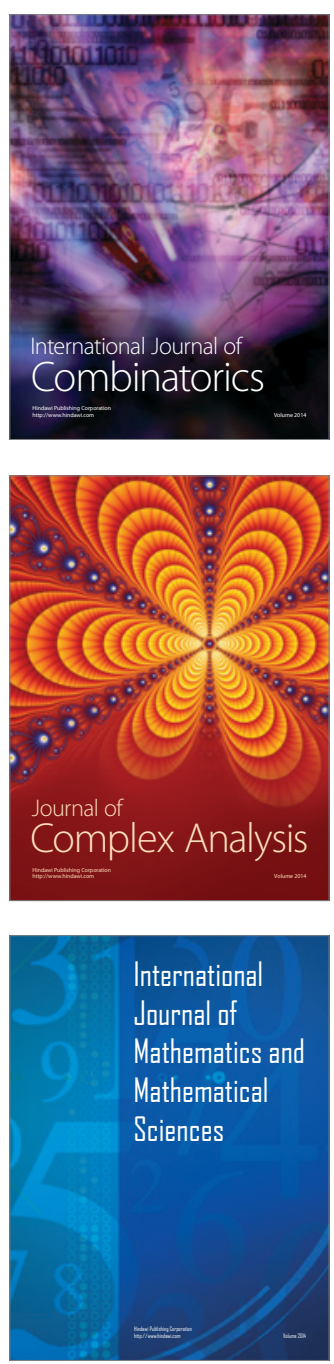
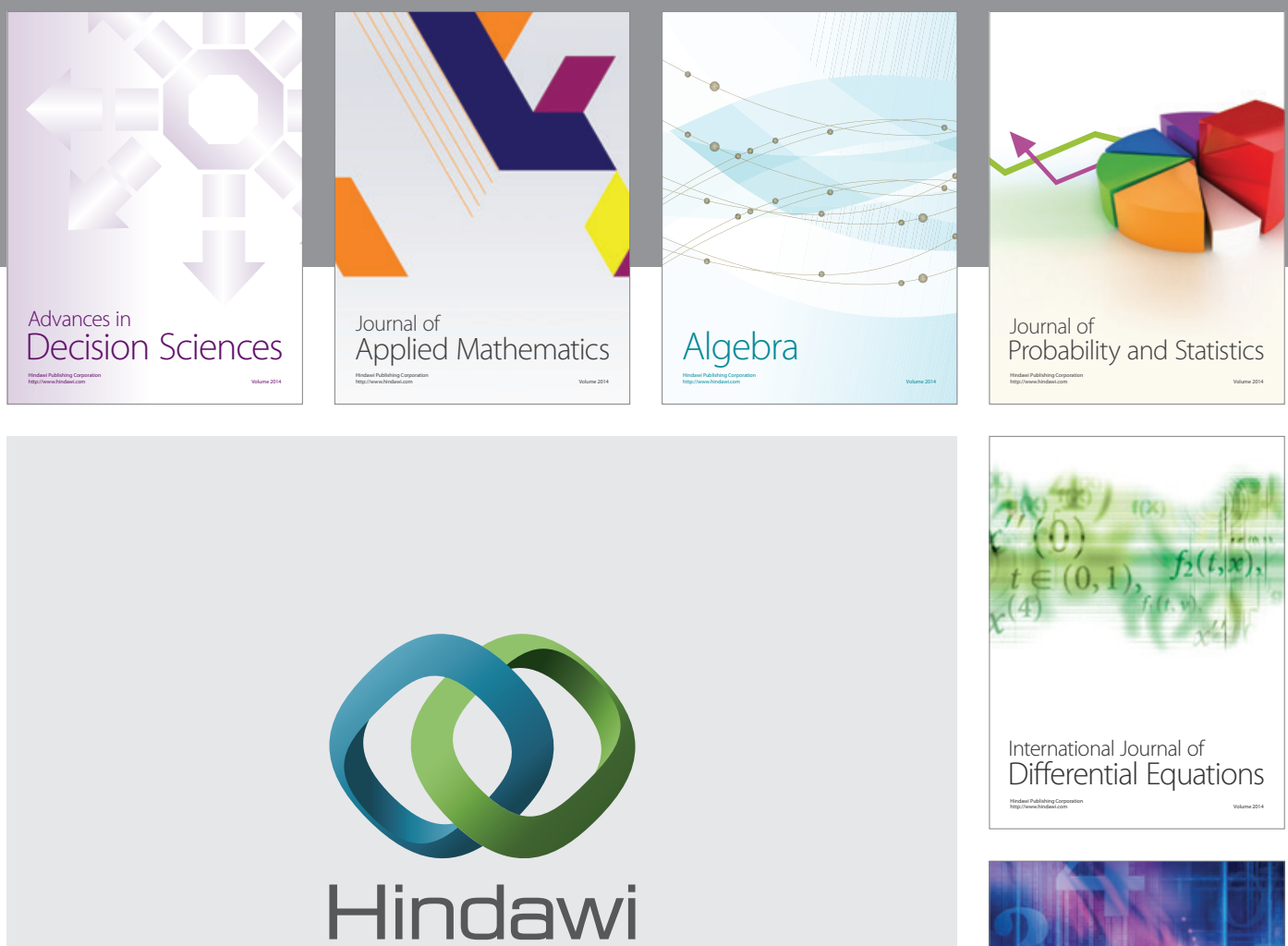

Submit your manuscripts at http://www.hindawi.com
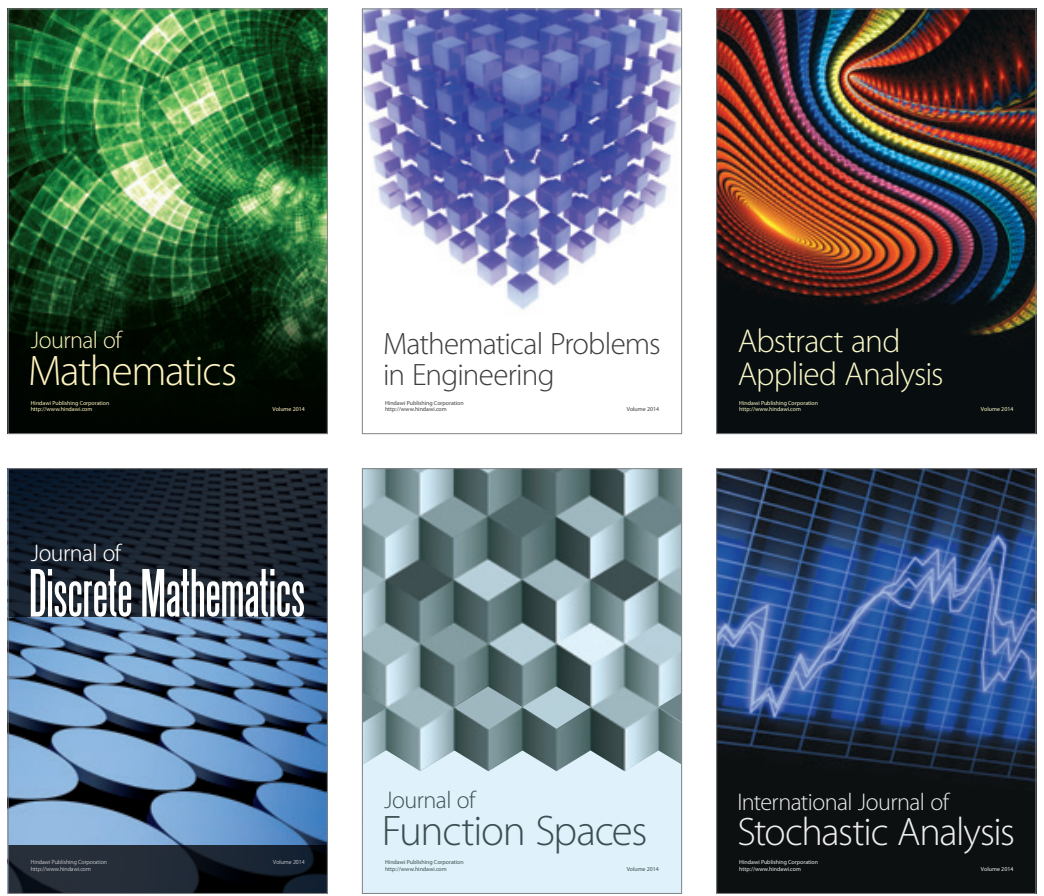

Journal of

Function Spaces

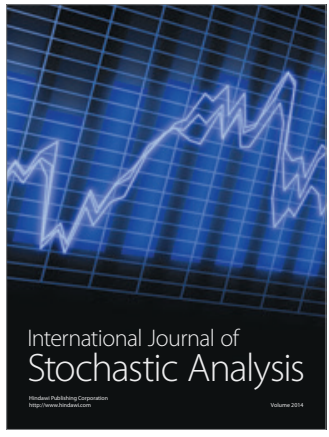

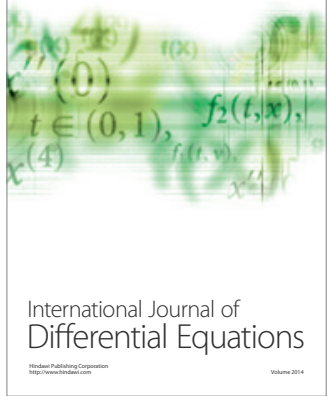
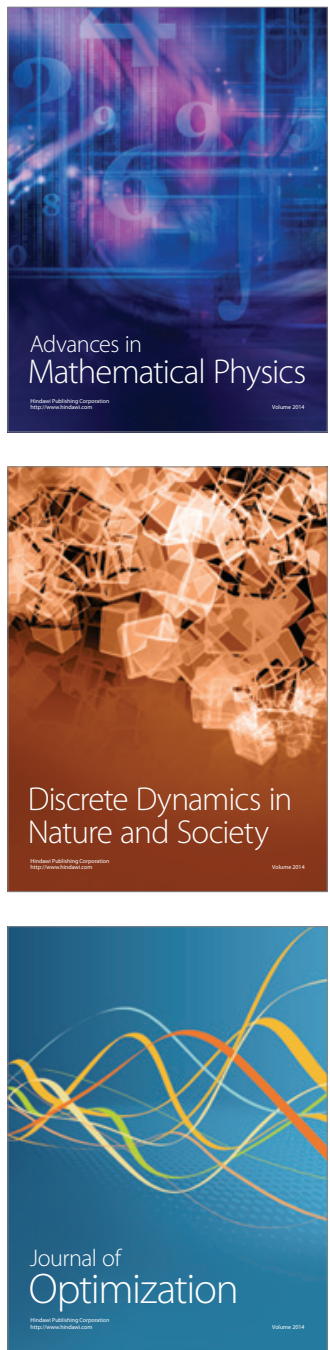